\title{
Correlation-driven Lifshitz transition at the emergence of the pseudogap phase in the two-dimensional Hubbard model
}

\author{
Helena Bragança, ${ }^{1,2}$ Shiro Sakai, ${ }^{3}$ M. C. O. Aguiar, ${ }^{1}$ and Marcello Civelli ${ }^{2}$ \\ ${ }^{I}$ Departamento de Física, Universidade Federal de Minas Gerais, C. P. 702, 30123-970, Belo Horizonte, MG, Brazil \\ ${ }^{2}$ Laboratoire de Physique des Solides, Univ. Paris-Sud, \\ Université Paris-Saclay, CNRS UMR 8502, F-91405 Orsay Cedex, France \\ ${ }^{3}$ Center for Emergent Matter Science, RIKEN, Wako, Saitama 351-0198, Japan
}

(Dated: January 22, 2018)

\begin{abstract}
We study the relationship between the pseudogap and Fermi-surface topology in the two-dimensional Hubbard model by means of the cellular dynamical mean-field theory. We find two possible mean-field metallic solutions on a broad range of interaction, doping and frustration: a conventional renormalized metal and an unconventional pseudogap metal. At half-filling, the conventional metal is more stable and displays an interactiondriven Mott metal-insulator transition. However, for large interaction and small doping, region that is relevant for cuprates, the pseudogap phase becomes the ground state. By increasing doping, we show that a first-order transition from the pseudogap to the conventional metal is tight to a change of the Fermi surface from hole to electron like, unveiling a correlation-driven mechanism for a Lifshitz transition. This explains the puzzling link between pseudogap phase and Fermi surface topology which has been pointed out in recent experiments.
\end{abstract}

In order to understand superconductivity [1-3] one must first understand the normal metallic state, appearing above a critical temperature $\left(T_{c}\right)$, from which it takes its roots. The high- $T_{c}$ superconductivity in cuprates remains unsolved, mainly because its normal metallic state, the pseudogap (PG) phase, has not been well understood. It has been therefore a central issue to establish the origin of the pseudogap and its relation with the high- $T_{c}$ superconducting mechanism [4]. The PG has been revealed [5, 6] in spectroscopic responses [7] and thermodynamic and transport properties [8] by a loss of spectral weight, which departs from the conventional Fermi liquid (FL) theory of metals [9, 10]. Recent experiments have pointed out that when a Lifshitz transition [i.e. a change of Fermi Surface (FS) topology from electron-like (e) to hole-like (h)] is tuned in the PG phase of a cuprate material, the PG ends abruptly. This takes place for instance at a doping $p^{*}$ on the overdoped region of $\mathrm{Bi}_{2} \mathrm{Sr}_{2} \mathrm{CuO}_{6+\delta}$ [11], $\mathrm{Bi}_{2} \mathrm{Sr}_{2} \mathrm{CaCu}_{2} \mathrm{O}_{8+\delta}$ [12, 13] and in $\mathrm{La}_{1.6-x} \mathrm{Nd}_{0.4} \mathrm{Sr}_{x} \mathrm{CuO}_{4}[14]$. This finding has been strongly debated, as it challenges our current understanding of the PG phase [4] and its relation with superconductivity

Here we give a rational explanation to all these observations within the framework of the two-dimensional Hubbard model solved with the cellular dynamical mean field theory (CDMFT) [15-17]. We first show that two metallic solutions exist: a rather regular correlated Fermi-liquid metal (CFM), and a PG metal (PGM), which violates Fermi liquid theory, by developing a pole-divergence in the self-energy. This result could account for contradicting reports about the existence of the Mott metal-insulator transition (MIT) at half-filling (zero doping) in two dimensions. The PGM is metastable at weak interactions, having higher energy than the CFM. However, by increasing interaction at low doping (region relevant for underdoped cuprates) the PGM emerges as the stable phase, up to the doping value $p^{*}$. This is consistent with the CDMFT results of Sordi et al.[18]. Most importantly, we show that the PGM is bound to have always a h-FS. The CFM instead can undergo a Lifshitz transition at a doping $p_{l t}$. However, for strong interaction the CFM is stable only for doping $p>p_{l t}$, i.e. it has always an e-FS. Hence the transition from the PGM to the CFM at $p^{*}$ is accompanied by a corresponding change from a h-FS to an e-FS, unveiling a novel correlationdriven mechanism of the Lifshitz transition. These results explain why the PG must sharply end when a Lifshitz transition occurs [11], or is tuned by pressure [14], in cuprates.

We consider the two-dimensional one-band Hubbard model:

$$
\mathcal{H}=-\sum_{\mathbf{k} \sigma} \xi_{\mathbf{k}} c_{\mathbf{k} \sigma}^{\dagger} c_{\mathbf{k} \sigma}+U \sum_{i} n_{i \uparrow} n_{i \downarrow}
$$

where $c_{\mathbf{k} \sigma}=(1 / \sqrt{L}) \sum_{i} \exp \left(-i \mathbf{k} \cdot \mathbf{r}_{i}\right) c_{i, \sigma}$ destroys an electron with spin $\sigma$ and momentum $\mathbf{k}, n_{i \sigma}=c_{i \sigma}^{\dagger} c_{i \sigma}$ is the density operator on site $i$ of a $L$-site square lattice. $\xi_{\mathbf{k}}=-2 t\left(\cos k_{x}+\right.$ $\left.\cos k_{y}\right)-4 t^{\prime} \cos k_{x} \cos k_{y}-\mu$, where $t\left(t^{\prime}\right)$ is (next) nearestneighbor-site hopping integral, $\mu$ the chemical potential controlling the doping level $p=1-(1 / L) \sum_{i, \sigma}\left\langle n_{i \sigma}\right\rangle$. We implement the CDMFT at zero temperature $(T=0)$ using Lanczos. This maps $\mathcal{H}$ onto a $2 \times 2$ cluster coupled to an 8 -site bath [1921] [see section I of the Supplemental Material (SM)]. The numerical calculation provides the frequency dependent Green's function in the corner points of the first quadrant of the Brillouin zone (BZ). To obtain the lattice quantities in momentum space we perform a periodization based on the cumulant [2224]. We calculate the total energy as described in Ref. [25] and in section II of the SM, which includes Ref. [26]. We set $t=1$ and explore the paramagnetic phase diagram in the $U-t^{\prime}$ space at half-filling (Fig. 11), and the $U-p$ space at fixed $t^{\prime}=-0.1$ (Fig. 1p). At $T=0$, the ground state is broken symmetry phase: antiferromagnetism at half-filling and small doping, and superconductivity upon doping. These phases have been widely studied within CDMFT [16-18, 27. 32]. Here we focus on the paramagnetic solution which, albeit being the normal-state ground-state only at $T>T_{c}$, is the mean-field phase from which broken orders take roots. This 

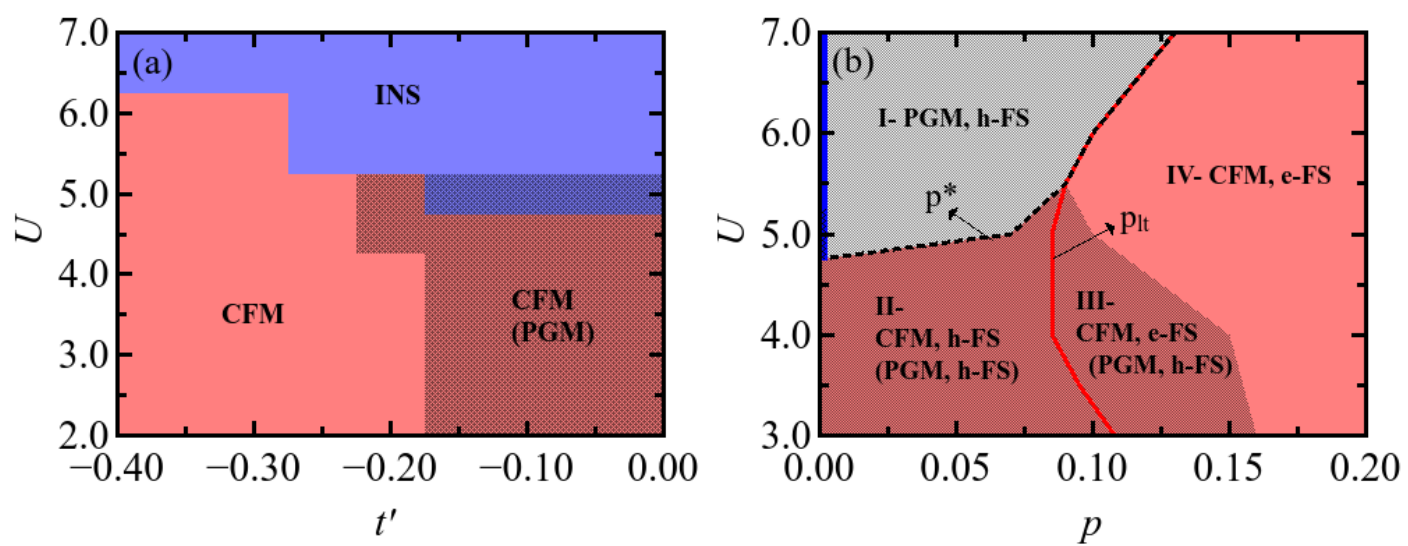

FIG. 1: (a) $U-t^{\prime}$ phase diagram for the normal state of the two-dimensional Hubbard model at half-filling $(p=0)$. The blue, pink and dark regions bound respectively a Mott insulator (INS), a correlated Fermi-liquid metal (CFM) and a coexisting pseudogap metal (PGM). The CFM has always lower energy than the PGM (in parenthesis). (b) $U-p$ phase diagram with $t^{\prime}=-0.1$. The blue vertical line at $p=0$ is the INS. The continuous red line $p_{l t}$ marks a Lifshitz transition, where the FS topology changes from electron-like (e-FS) to hole-like (h-FS). The dashed black line $p^{*}$ marks the boundary where the PGM energy becomes higher than the CFM one.

allows us to study the FS topology and its relation with the pseudogap.

We start with the half-filled system [Fig. 11(a)]. A relevant question is whether in two dimensions a gap is present in the paramagnetic solution at any small $U$, like in one dimension [33] and as it was proposed by P.W. Anderson [34], or whether the system becomes a regular metal under a critical $U_{c}$, i.e. there is a Mott MIT, like in infinite dimensions [35]. This issue has been considered by various groups using quantum cluster methods [36-41], but it has not been completely clarified. In these studies it was considered the particle-hole symmetric $t^{\prime}=0$ case, which is especially singular because a $\mathbf{k}=(\pi, \pi)$ nesting vector acts on the whole FS producing divergent susceptibilities. It is very likely then that at $T=0$ a gap always opens in the system. To verify Anderson's conjecture we have considered $t^{\prime} \neq 0$. Our main result is that, for $U<U_{c}$, we find two different metallic solutions, the CFM and the PGM, as it is shown in Fig. 11a). The PGM coexists with the CFM for a broad range of $U$ and $t^{\prime}$ values, disappearing only for large $\left|t^{\prime}\right|$. For interaction greater than $U \simeq 5 t$ we recover the well known Mott insulating phase. We shall now show that the CFM is the FL solution displaying the Mott transition, in agreement with the statements of publications [36-38, 41], while the PGM solution presents always a gap in the spectra reminiscent of the solution found in the works of Ref. [39, 40].

To this purpose, we set $t^{\prime}=-0.1$ and display in Fig. 2 the spectral function $A(\mathbf{k}, \omega)=-\frac{1}{\pi} \operatorname{Im} G(\mathbf{k}, \omega)$ and the imaginary part of the self-energy $\operatorname{Im} \Sigma(\mathbf{k}, \omega)$ at $\mathbf{k}=(0, \pi)$, close to the Fermi level $(|\omega|<0.8 t$ ) (see SM section III for a broader $\omega$-range including the Hubbard bands). The CFM (red-dotted curve) displays typical features of a FL: finite spectral peak at the Fermi level $\omega=0$ (Fig. 2 a) and $\sim \omega^{2}$ behavior in $\operatorname{Im} \Sigma$ (Fig. 2 b). The PGM (black-solid curve) displays sharply distinct features. $A(\mathbf{k}, \omega)$ has a minimum at $\omega=0$ (Fig. 22a),

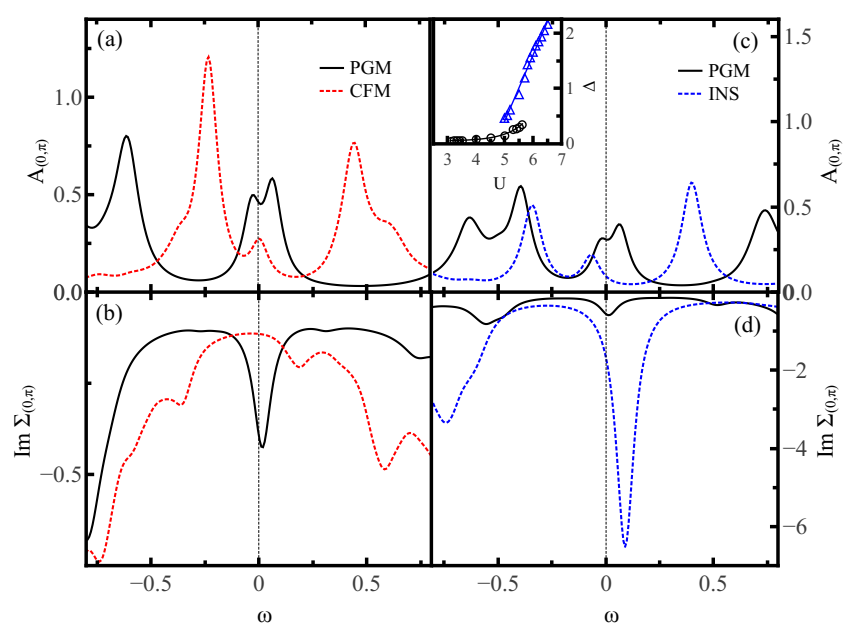

FIG. 2: Low-energy spectral function $A(\mathbf{k}, \omega)$ (a) at $\mathbf{k}=(0, \pi)$ and corresponding imaginary part of the self-energy $\operatorname{Im} \Sigma(\mathbf{k}, \omega)$ (b) of the two coexisting CFM and PGM at half-filling, $U=4.0, t^{\prime}=-0.1$. Comparison of $A(\mathbf{k}, \omega)$ (c) and $\operatorname{Im} \Sigma(\mathbf{k}, \omega)$ (d) between the PGM and the Mott insulator for $U=5.0, t^{\prime}=-0.1$. Inset: spectral gap of $A(\mathbf{k}, \omega)$ as a function of $U$ for the PGM (circles) and the Mott insulator (triangles).

showing a small gap $\Delta$, which we plot in the inset of Fig. 22(c) (circles) as a function of $U$ together with the insulator gap (triangles). $\operatorname{Im} \Sigma$ displays a pole-like divergence (Fig. 2 b), which breaks the FL. This behavior of the self-energy is similar to what is expected in a Mott insulator (blue-dotted curve in Fig. 2 d), whose gap is always characterized by a pole in the self-energy, though in the PGM the intensity of the divergence is reduced and the two solutions are not smoothly connected, showing a coexistence region (inset of Fig. 2 2 c).

We find that at half-filling and different $t^{\prime}$ the CFM al- 


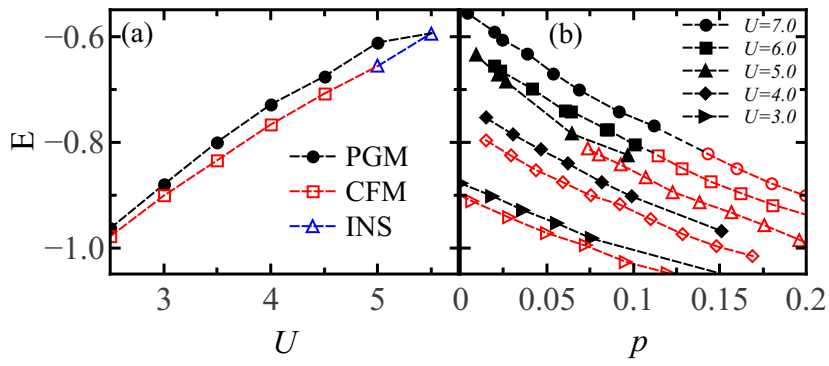

FIG. 3: Total energy E (a) as a function of interaction at half-filling and (b) as a function of doping, both for $t^{\prime}=-0.1$. Red-open symbols correspond to the CFM solution, while black-filled ones represent the PGM.

ways shows a lower energy than the PGM, as displayed in Fig. 3(a). This validates the concept of Mott MIT also in the two-dimensional Hubbard model (see SM for the kinetic and potential energies). The unstable PGM remains however reminiscent of Anderson's RVB theory [34, 42], though relevant differences have been already pointed out [43].

The PGM can become however the relevant lowest-energy phase upon doping. In the $U-p$ phase diagram of Fig. 1 b), where $t^{\prime}=-0.1$, we can clearly identify three regions: The PGM (I), the CFM (IV) and a coexisting CFM-PGM region (II and III). In this latter case (see Fig. 3 b for $U=3,4$ ), the CFM has always the lowest total energy. For $U>U_{c} \simeq 5 t$, however, at small doping (region relevant for underdoped cuprates) the PGM emerges as the stable solution, while the CFM persists at high dopings. These results are consistent with those of ref. [18, 44] which shows a small first-order coexistence region between regions I and IV, which closes at a tricritical point and become continuous for $U \geq 7 t$. We think that our Lanczos-implementation has difficulties to enter into this small coexistence region, and we cannot say if the transition becomes second order for $U \geq 7 t$. However the first-order character of the transition in the region that we considered is shown by the fact that the PGM and the CFM are not smoothly connected.

We confirm in the doped system the key physical properties differentiating the CFM and PGM phases, established for halffilling. Namely the PGM always breaks the FL displaying a peak in $\operatorname{Im} \Sigma$ (see Fig. 44,c,d), which has now slightly moved to positive frequency. On the other hand the CFM phase is FL-like on all the phase diagram, displaying a well behaved $\omega^{2}$ dependence of $\operatorname{Im} \Sigma$ (Fig. 4 b,c,d).

Let us now discuss the implications of these findings in the context of the PG phase of cuprates and its relation with the FS topology. This has been the subject of pioneering studies [18, 22, 29, 30, 45-51], though the physical mechanism at the origin of this relation has remained not well clarified. The first crucial observation is that the pole in the self-energy in the PGM solution strongly enhances the scattering in the neighborhood of $\mathbf{k}=(0, \pi)$ (antinodes in cuprates). As a consequence, the spectral weight on the FS around $\mathbf{k}=(0, \pi)$
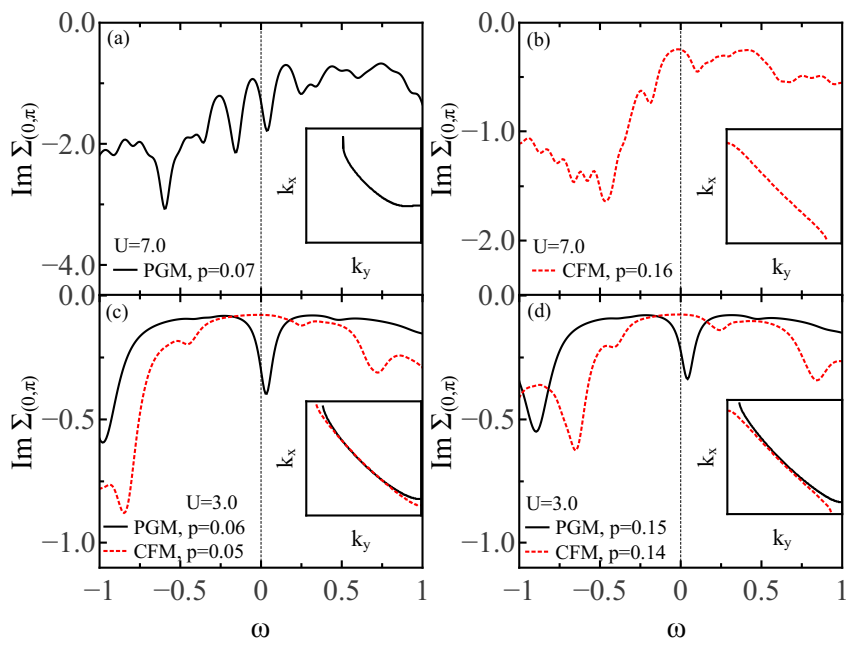

FIG. 4: Comparison of the imaginary part of the self-energy at the antinodal point $\mathbf{k}=(0, \pi)$, between CFM and PGM solutions in various regions [(a) region I, (b) IV, (c) II, (d) III ] of the $U-p$ phase diagram of Fig. 11. Inset displays the corresponding FS in the first quadrant of the $\mathrm{BZ}\left(k_{x}, k_{y} \in[0, \pi]\right)$.

is strongly suppressed, giving origin to the well known break of the FS into arcs. This can be shown in the spectral function $A(\mathbf{k}, \omega=0)$ plotted in Fig. 5(a),(c). These results are consistent with previous CDMFT studies [29, 46, 52] and well describe the angle-resolved photoemission spectroscopy measurements on cuprates [7]. The CFM does not show any Fermi $\operatorname{arc}($ Fig. 5. b,d), rather the spectral intensity is enhanced at the antinodes because of the proximity to a van Hove singularity.

The second crucial observation (insets of Fig. (4) is that the $P G M$ solution has always a $h-F S$. To get some insight into this issue, we notice that in the PGM the low-frequency pole in the self-energy can be well described by $\Sigma(\mathbf{k}, \omega) \simeq V^{2} /(\omega-$ $\left.\xi_{\mathrm{k}}^{f}\right)$. This expression has been derived in the framework of a low-energy model called "hidden fermion" [43, 53], but also proposed by complementary approaches to the PG phase of cuprates [47, 54, 56]. $\xi_{\mathrm{k}}^{f}$ is located slightly above the Fermi energy in the antinodal region. We see then that, if $\mathbf{k}_{F}$ is the Fermi wave-vector of the non-interacting system located on the $(0, \pi)-(\pi, \pi)$ side of the BZ, such that $\xi_{\mathbf{k}_{F}}=0$, the pole-like singularity of the self-energy in the interacting system acts in such a way that the new Fermi wave-vector $\mathbf{k}_{F}^{\prime}$ is given by $\xi_{\mathbf{k}_{F}^{\prime}}-V^{2} / \xi_{\mathbf{k}_{F}^{\prime}}^{f}=0$. If $\xi_{\mathbf{k}_{F}^{\prime}}^{f}$ is positive as it is for $\mathbf{k}=(0, \pi)$, it acts as an enhanced chemical potential (see SM section IV) and $\mathbf{k}_{F}^{\prime}>\mathbf{k}_{F}$, i.e. the interacting FS is more hole-like. To undergo a transition from the h-FS to e-FS in the PGM, the FS must cross the $\mathbf{k}=(0, \pi)$ point. But this is pre-empted by the pole-singularity of the self-energy. One possibility is to have the pole singularity position $\xi_{\mathbf{k}}^{f}$ move to negative frequencies. As noticed in Ref. [48], this can be achieved by tuning $t^{\prime}$ to positive values, which is equivalent to consider electron-doped cuprates, as the same tuning can be realized by a particle-hole transformation of the Hamiltonian 

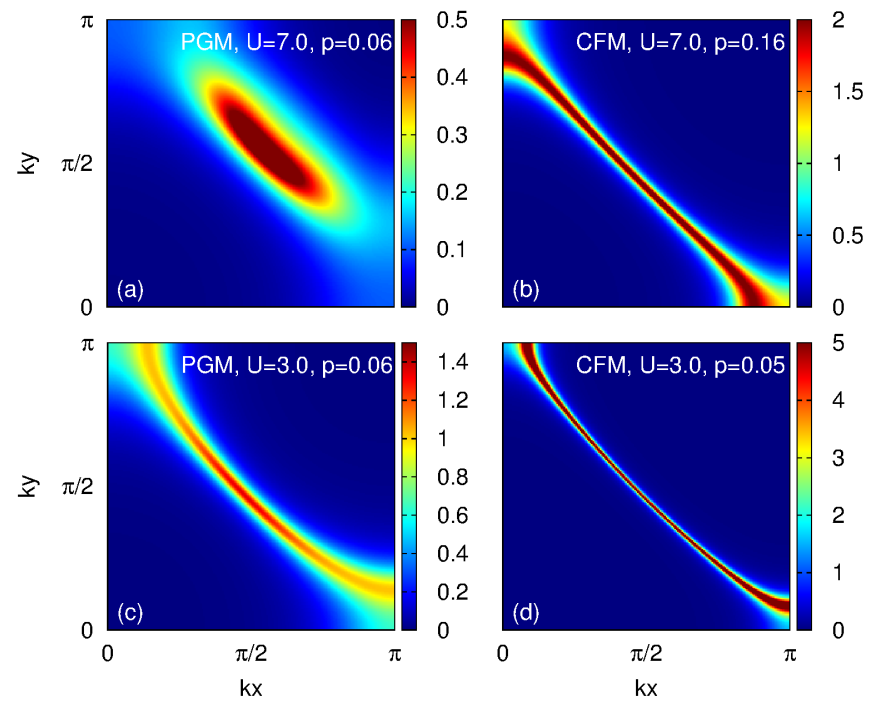

FIG. 5: Spectral function $A(\mathbf{k}, \omega=0)$ in the first quadrant of the $\mathrm{BZ}$ for the CFM and the PGM. At weak interaction $U=3$ and small doping $p=0.05$, the underlying FS is hole-like in both solutions. In this case however the CFM is the stable solution, not showing Fermi arcs. At strong interaction $U=7.0$, the PGM is stable at small doping ( $p=0.06$ ), displaying Fermi arcs and a h-FS, while the CFM is stable at high doping $(p=0.16)$, displaying no arc and an e-FS.

(1) that changes the sign of $t^{\prime}$ [52]. Another possibility is to have the pole singularity disappear $(V \rightarrow 0)$, but in this case one loses the PGM solution. We establish then an indissoluble tight relationship within the PGM solution between pole in the self-energy, PG, and hole-like FS.

Putting all together, we provide a rational understanding to the experimental observations that the PG ending point is linked to a Lifshitz transition, and above all why this appears first-order [12, 13] or at least very sharp [11, 14] in experiments. Starting from weak $U$ [see Fig. 11.b)] the stable solution is the CFM, which by increasing doping presents a renormalized [48] Fermi-liquid-like Lifshitz transition at $p=$ $p_{l t}$ (continuous red line in Fig. 11.b), where one goes continuously from a h-FS to an e-FS. There is no PG in this case; the PG is present in the PGM, which has a h-FS (Fig. 5.c), but this phase is unstable. When the $p_{l t}$ boundary on the $U-p$ phase diagram meets the PGM-CFM transition boundary $p^{*}$ (black-dashed lines) at stronger $U \simeq 5.5 t$, we find for $p<p^{*}$ the PGM as the stable solution, which has a h-FS, while for $p>p^{*}$ the CFM is the stable solution, but this has already an e-FS (Fig. 5 b). By increasing $p$ then the change from h-FS to e-FS is bound to the PGM-CFM phase transition $\left(p_{l t} \equiv p^{*}\right)$, which is first order, providing a correlated mechanism for the Lifshitz transition. This is consistent with the experimental observations in Bi-based [11-13] and Labased compounds[14]. On the other hand, there is a region of the phase diagram in the range $4.5 t<U<5.5 t$ where the PGM-CFM transition takes place at a doping smaller than the Lifshitz transition of the CFM solution, i.e. $p^{*}<p_{l t}$. At $p^{*}$ the PG disappears but the FS is still hole-like. This may account for the Tl-based [57, 58] and Y-based[59] cuprates, which have been reported to have a h-FS but no PG. This may also crucially depend on the $\left|t^{\prime} / t\right|$ value, as pointed out in Ref.[48]. Our paramagnetic CDMFT phase-diagram of the two-dimensional Hubbard model can then fully account for apparently contradicting and debated experimental results on different members of the cuprate family, showing that there truly exists a tight relationship between PG and FS topology. This should manifests itself in cuprates whenever a Lifshitz transition takes place in the pseudogap phase.

In conclusion, we have studied the paramagnetic normalstate of the two-dimensional Hubbard model at zero temperature for a broad range of interaction, $U$, frustration, $t^{\prime}$, and doping level, $p$. Our main finding is the coexistence of a correlated Fermi liquid metal (CFM) with a non-Fermi liquid metal (PGM). At half-filling, we answer to an open debate by showing that the CFM is the stable solution and displays a correlation-driven Mott MIT, differently from the PGM which displays instead a PG in the spectra. Next we show that for strong interactions and small doping, region relevant for underdoped cuprates, the PGM becomes the stable solution. This result is at the origin of a correlation-driven Lifshitz transition, as by increasing doping a first-order transition takes place from the PGM phase, which has a h-FS, to the CFM, which at this interaction values has an e-FS. Our theory rationalizes the variety displayed on the phase diagram of the cuprate family, telling us that if the PG meets a Lifshitz transition, then it should collapse. This implies also that the PG cannot exists on an e-FS. The behavior of the PG that is sensitive to the FS topology must be contrasted with the one of superconductivity, which does not appear much affected by the Lifshitz transition[13]. This may represent the key to finally unveil the true nature of the relation between the PG and the high- $T_{c}$ mechanism. Future experimental and theoretical investigations should be pursued in this direction.

During our investigations, we became aware of the work of Ref. [48], whose results are in good agreement with ours. Our PGM, however, is found as a second metastable solution distinct from the CFM. This in particular discloses the origin of the tight link between the PG and correlated first-order Lifshitz transition.

We thank I. Paul for his insightful comments. We acknowledge discussion with W. Wu, M. Ferrero, A. Georges, B. Loret, A. Sacuto, O. Parcollet, A.-M. Tremblay, G. Sordi. This work was supported by CNPq, CAPES and FAPEMIG, and grants JSPS KAKENHI (16H06345 and 17K14350), support from the INCT on Quantum Information/CNPq is also gratefully acknowledged. Part of the results were obtained at CENAPAD-SP. 
[1] J. Bardeen, L. N. Cooper, J. R. Schrieffer, Microscopic Theory of Superconductivity, Phys. Rev. 106 (1), 162164 (1957).

[2] A.B. Migdal, Interaction between Electrons and Lattice Vibrations in a Normal Metal, JETP 7, No. 6, 996 (1958).

[3] G.M. Eliashberg, Interactions between Electrons and Lattice Vibrations in a Superconductor, JETP 11, No. 3, 696 (1960).

[4] M. Norman, D. Pines and C. Kallin, The pseudogap: friend or foe of high $T_{c}$ ?, Advances in Physics 54, 715733 (2005).

[5] H. Alloul, T. Ohno, and P. Mendels, ${ }^{89} Y$ NMR evidence for a fermi-liquid behavior in $\mathrm{YBa}_{2} \mathrm{Cu}_{3} \mathrm{O}_{6+x}$, Phys. Rev. Lett. 63, 1700 (1989).

[6] W. W. Warren, R. E. Walstedt, G. F. Brennert, R. J. Cava, R. Tycko, R. F. Bell, and G. Dabbagh, Cu spin dynamics and superconducting precursor effects in planes above Tc in $\mathrm{YBa}_{2} \mathrm{Cu}_{3} \mathrm{O}_{6.7}$, Phys. Rev. Lett. 62, 1193 (1989).

[7] Andrea Damascelli, Zahid Hussain, and Zhi-Xun Shen, Angleresolved photoemission studies of the cuprate superconductors, Rev. Mod. Phys. 75, 473 (2003).

[8] T. Timusk and B. Statt, The pseudogap in high-temperature superconductors: an experimental survey, Reports on Progress in Physics 62 (1): 61122 (1999).

[9] A. A. Abrikosov, L. P. Gorkov, and I. E. Dzyaloshinski, Methods of Quantum Field Theory in Statistical Mechanics, (Dover, New York, 1963).

[10] P. Nozières, Interacting Fermi Systems, (Benjamin, New York, 1964).

[11] A. Piriou, N. Jenkins, C. Berthod, I. Maggio-Aprile, and O. Fischer, First direct observation of the Van Hove singularity in the tunnelling spectra of cuprates, Nat. Commun. 2, 221 (2011).

[12] S. Benhabib, A. Sacuto, M. Civelli, I. Paul, M. Cazayous, Y. Gallais, M.-A. Measson, R. D. Zhong, J. Schneeloch, G. D. Gu, D. Colson, A. Forget, Collapse of the Normal State Pseudogap at a Lifshitz Transition in $\mathrm{Bi}_{2} \mathrm{Sr}_{2} \mathrm{CaCu}_{2} \mathrm{O}_{8+\delta}$ Cuprate Superconductor, Phys. Rev. Lett. 114, 147001, (2015).

[13] B. Loret, S. Sakai, S. Benhabib, Y. Gallais, M. Cazayous, M. A. Measson, R. D. Zhong, J. Schneeloch, G. D. Gu, A. Forget, D. Colson, I. Paul, M. Civelli, A. Sacuto, Vertical temperatureboundary of the pseudogap under the superconducting dome of the $\mathrm{Bi}_{2} \mathrm{Sr}_{2} \mathrm{CaCu}_{2} \mathrm{O}_{8+d}$ phase-diagram, arXiv:1703.00794

[14] N. Doiron-Leyraud, O. Cyr-Choinière, S. Badoux, A. Ataei, C. Collignon, A. Gourgout, S. Dufour-Beauséjour, F. F. Tafti, F. Laliberté, M. Matusiak, D. Graf, M. Kim, J.-S. Zhou, N. Momono, T. Kurosawa, H. Takagi, and Louis Taillefer, public communications, to appear.

[15] G. Kotliar, S. Y. Savrasov, G. Pálsson, G. Biroli, Cellular dynamical mean field approach to strongly correlated systems, Phys. Rev. Lett. 87, 186401 (2001).

[16] T. Maier, M. Jarrell, T. Pruschke, and M. H. Hettler, Quantum cluster theories, Rev. Mod. Phys. 77, 1027 (2005).

[17] A.-M. S. Tremblay, B. Kyung, and D. Sénéchal, Pseudogap and high-temperature superconductivity from weak to strong coupling. Towards a quantitative theory. Low Temperature Physics 32, 424 (2006).

[18] G. Sordi, K. Haule, and A.-M. S. Tremblay, Finite Doping Signatures of the Mott Transition in the Two-Dimensional Hubbard Model, Phys. Rev. Lett. 104, 226402 (2010).

[19] M. Civelli, The doping-driven evolution of the superconducting state of a doped Mott insulator: a key for the high temperature superconductivity, Phys. Rev. B 79, 195113 (2009).

[20] M. Capone, M. Civelli, S. S. Kancharla, C. Castellani, and G. Kotliar, Cluster-dynamical mean-field theory of the density- driven Mott transition in the one-dimensional Hubbard model, Phys. Rev. B 69, 195105 (2004).

[21] Marcello Civelli, Investigation of Strongly Correlated Electron Systems with Cellular Dynamical Mean Field Theory, PhD Thesis, Rutgers University, Piscataway, New Jersey, USA (2006), arXiv:0710.2802.

[22] Tudor D. Stanescu and Gabriel Kotliar, Fermi arcs and hidden zeros of the Green function in the pseudogap state, Phys. Rev. B 74, 125110 (2006).

[23] Tudor D. Stanescu, Marcello Civelli, Kristjan Haule, Gabriel Kotliar, A cellular dynamical mean field theory approach to Mottness, Ann. Phys. (N.Y.) 321, 1682 (2006).

[24] Shiro Sakai, Giorgio Sangiovanni, Marcello Civelli, Yukitoshi Motome, Karsten Held, Masatoshi Imada, Cluster-size dependence in cellular dynamical mean-field theory, Phys. Rev. B 85, 035102 (2012).

[25] E. Gull, A. J. Millis, Energetics of superconductivity in the two dimensional Hubbard model, Phys. Rev. B 86, 241106(R) (2012).

[26] Gerald D. Mahan, Many-Particle Physics, 3rd ed., Kluwer Academic/Plenum Publishers, New York, 2000.

[27] G. Kotliar, S. Y. Savrasov, K. Haule, V. S. Oudovenko, O. Parcollet, and C. A. Marianetti, Electronic structure calculations with dynamical mean-field theory, Rev. Mod. Phys. 78, 865 (2006).

[28] S. S. Kancharla, B. Kyung, D. Sénéchal, M. Civelli, M. Capone, G. Kotliar, and A.-M. S. Tremblay, Anomalous superconductivity and its competition with antiferromagnetism in doped Mott insulators, Phys. Rev. B 77, 184516 (2008).

[29] Michel Ferrero, Pablo S. Cornaglia, Lorenzo De Leo, Olivier Parcollet, Gabriel Kotliar, and Antoine Georges, Pseudogap opening and formation of Fermi arcs as an orbital-selective Mott transition in momentum space, Phys. Rev. B 80, 064501 (2009).

[30] E. Gull, M. Ferrero, O. Parcollet, A. Georges, and A. J. Millis, Momentum space anisotropy and pseudogaps: a comparative cluster dynamical mean field analysis of the dopingdriven metal-insulator transition in the two dimensional Hubbard model, Phys. Rev. B 82, 155101 (2010).

[31] E. Gull, O. Parcollet, A. J. Millis, Superconductivity and the Pseudogap in the two-dimensional Hubbard model, Phys. Rev. Lett. 110, 216405 (2013).

[32] E. Gull and A. J. Millis, Quasiparticle Properties of the Superconducting State of the Two Dimensional Hubbard Model, Phys. Rev. B 91, 085116 (2015).

[33] E. H. Lieb and F. Y. Wu, Absence of the Mott transition in an exact solution of the short-range, one-band model in one dimension, Phys. Rev. Lett. 25, 9 (1968).

[34] P. W. Anderson, The Theory of Superconductivity in the HighTc Cuprates, Princeton University Press, Princeton (1997).

[35] A. Georges and G. Kotliar, Hubbard model in infinite dimensions, Phys. Rev. B 45, 6479 (1992).

[36] Y. Z. Zhang, Masatoshi Imada, Pseudogap and Mott Transition Studied by Cellular Dynamical Mean Field Theory, Phys. Rev. B 76, 045108 (2007).

[37] H. Park, K. Haule, G. Kotliar, Cluster Dynamical Mean Field Theory of the Mott Transition, Phys. Rev. Lett. 101, 186403 (2008).

[38] Matthias Balzer, Bumsoo Kyung, David Sénéchal, A.-M. S. Tremblay, Michael Potthoff, First order Mott transition at zero temperature in two dimensions: Variational plaquette study, Europhys. Lett. 85, 17002 (2009).

[39] S. Moukouri and M. Jarrell, Absence of a Slater Transition in the Two-Dimensional Hubbard Model, Phys. Rev. Lett. 87, 
167010 (2001).

[40] T. Schäfer, F. Geles, D. Rost, G. Rohringer, E. Arrigoni, K. Held, N. Blümer, M. Aichhorn, and A. Toschi, Fate of the false Mott-Hubbard transition in two dimensions, Phys. Rev. B 91, 125109 (2015).

[41] L. Fratino, P. Sémon, M. Charlebois, G. Sordi, A.-M. S. Tremblay, Signatures of the Mott transition in the antiferromagnetic state of the two-dimensional Hubbard model, Phys. Rev. B 95, 235109 (2017).

[42] Luca F. Tocchio, Federico Becca, and Sandro Sorella, Hidden Mott transition and large- $U$ superconductivity in the twodimensional Hubbard model, Phys. Rev. B 94, 195126 (2016).

[43] Shiro Sakai, Marcello Civelli, Masatoshi Imada, Evidence for hidden fermion that triggers high-temperature superconductivity in cuprates, Phys. Rev. Lett. 116, 057003 (2016).

[44] Jean Paul Latyr Faye and David Sénéchal, Pseudogap to metal transition in the anisotropic two-dimensional Hubbard model, arXiv:1707.09446.

[45] Ansgar Liebsch and Ning-Hua Tong, Finite-temperature exact diagonalization cluster dynamical mean-field study of the twodimensional Hubbard model: Pseudogap, non-Fermi-liquid behavior, and particle-hole asymmetry, Phys. Rev. B 80, 165126 (2009).

[46] Shiro Sakai, Yukitoshi Motome, and Masatoshi Imada, Evolution of Electronic Structure of Doped Mott Insulators: Reconstruction of Poles and Zeros of Greens Function, Phys. Rev. Lett. 102, 056404 (2009).

[47] Kai-Yu Yang, T. M. Rice and Fu-Chun Zhang, A Phenomenological Theory of The Pseudogap State, Phys. Rev. B 73, 174501 (2006).

[48] Wei Wu, Antoine Georges, Michel Ferrero, Pseudogap and Fermi surface topology in the two-dimensional Hubbard model, arXiv: 1707.06602 .

[49] K.-S. Chen, Z. Y. Meng, T. Pruschke, J. Moreno and M. Jarrell, Lifshitz transition in the two-dimensional Hubbard model,
Phys. Rev. B 86, 165136 (2012).

[50] David Sénéchal and A.-M. S. Tremblay, Hot Spots and Pseudogaps for Hole- and Electron-Doped High-Temperature Superconductors, Phys. Rev. Lett. 92, 126401 (2004).

[51] Th. A. Maier, Th. Pruschke, and M. Jarrell, Angle-resolved photoemission spectra of the Hubbard model, Phys. Rev. B 66, 075102 (2002).

[52] M. Civelli, M. Capone, S. S. Kancharla, O. Parcollet, and G. Kotliar, Dynamical Breakup of the Fermi Surface in a Doped Mott Insulator, Phys. Rev. Lett. 95, 106402 (2005).

[53] M. Imada, Y. Yamaji, S. Sakai and Y. Motome, Theory of pseudogap and superconductivity in doped Mott insulators, Ann. Phys. 523, 629 (2011).

[54] Y. Yamaji and M. Imada, Composite fermion theory for pseudogap phenomena and superconductivity in underdoped cuprate superconductors, Phys. Rev. B 83, 214522 (2011).

[55] Philip W. Phillips, Brandon W. Langley and Jimmy A. Hutasoit, Un-Fermi Liquids: Unparticles in Strongly Correlated Electron Matter, Phys. Rev. B 88, 115129 (2013).

[56] C. Morice, X. Montiel and C. Pépin, Evolution of spectral and transport quantities with doping in the $S U(2)$ theory of cuprates, arXiv: 1704.06557

[57] M. Platé, et al., Fermi surface and quasiparticle excitations of overdoped $\mathrm{Tl}_{2} \mathrm{Ba}_{2} \mathrm{CuO}_{6+\delta}$, Phys. Rev. Lett. 95, 077001 (2005).

[58] D.C. Peets, J.D.F. Mottershead, B. Wu, I.S. Elfimov, R. Liang, W.N. Hardy, D.A. Bonn, M. Raudsepp, N.J.C. Ingle, A Damascelli, $\mathrm{Tl}_{2} \mathrm{Ba}_{2} \mathrm{CuO}_{6+d}$ Brings Spectroscopic Probes Deep Into the Overdoped Regime of the High-Tc Cuprates, New Journal of Physics 9, 28 (2007).

[59] M.A. Hossain, J.D.F. Mottershead, A. Bostwick, J.L. McChesney, E. Rotenberg, R. Liang, W.N. Hardy, G.A. Sawatzky, I.S. Elfimov, D.A. Bonn and A. Damascelli, In situ doping control of the surface of high-temperature superconductors, Nature Physics 4, 527 (2008). 


\title{
Supplementary Material for \\ "Correlation-driven Lifshitz transition at the emergence of the pseudogap phase in the two-dimensional Hubbard Model"
}

\author{
Helena Bragança ${ }^{1,2}$, Shiro Sakai ${ }^{3}$, M. C. O. Aguiar ${ }^{1}$, Marcello Civelli $^{2}$ \\ ${ }^{1}$ Departamento de Física, Universidade Federal de Minas Gerais, C. P. 702, 30123-970, Belo Horizonte, MG, Brazil \\ ${ }^{2}$ Laboratoire de Physique des Solides, Univ. Paris-Sud, Université Paris-Saclay, CNRS UMR 8502, F-91405 Orsay Cedex, France \\ ${ }^{3}$ Center for Emergent Matter Science, RIKEN, Wako, Saitama 351-0198, Japan
}

\section{I - BATH DESCRIPTION WITHIN ED-CDMFT}

Within cellular dynamical mean field theory the Hubbard model (Eq. 11) is mapped onto a cluster Anderson model with $2 \times 2$ interacting sites surrounded by a non-interacting bath. The latter Hamiltonian is given by

$$
\begin{aligned}
H & =\sum_{i j}^{N_{c}} \sum_{\sigma} E_{i j \sigma} c_{i \sigma}^{\dagger} c_{j \sigma}+U \sum_{i}^{N_{c}} c_{i \uparrow}^{\dagger} c_{i \uparrow} c_{i \downarrow}^{\dagger} c_{i \downarrow} \\
& +\sum_{k} \sum_{\sigma} \varepsilon_{k \sigma} a_{k \sigma}^{\dagger} a_{k \sigma}+\sum_{l}^{N_{b}} \sum_{i \sigma}\left(V_{l i \sigma} a_{l \sigma}^{\dagger} c_{i \sigma}+\text { h.c. }\right)(1)
\end{aligned}
$$

$N_{c}=4$ for the $2 \times 2$ plaquette and the indexes $i$ and $j$ label the cluster sites. $c_{i \sigma}^{\dagger}$ and $a_{l \sigma}^{\dagger}$ create electrons with spin $\sigma$ on the cluster and the bath, respectively. The matrix elements $E_{i j \sigma}$ represent the hopping parameters of the original Hubbard model (i.e., $E_{i i}=\mu, E_{i, i+1}=t, E_{i, i+2}=t^{\prime}$ ) while the bath dispersion $\varepsilon_{k \sigma}$ and the cluster-bath hybridization $V_{l i \sigma}$ are self-consistently determined.

To solve the above Hamiltonian with exact diagonalization one have to truncate the bath to a finite number of sites, although the original Hubbard model is still in the thermodynamic limit. In this work we use $N_{b}=8$ bath sites, separated in two sub-baths with the same geometry of the cluster. In this case, the Anderson Hamiltonian can be represented as

$$
\begin{aligned}
H= & \sum_{\sigma} \Psi_{\sigma}^{\dagger} E_{\sigma} \Psi_{\sigma}+U \sum_{i} n_{i \uparrow} n_{i, \downarrow}+ \\
& +\sum_{\alpha \sigma}\left(\Phi_{\alpha \sigma}^{\dagger} E_{B \sigma}^{\alpha} \Phi_{\alpha \sigma}+\Phi_{\alpha \sigma}^{\dagger} V_{\alpha \sigma} \Psi_{\sigma}+\text { h.c. }\right)
\end{aligned}
$$

where $\alpha=1,2$ labels the sub-bath, $E_{\sigma}, E_{B \sigma}^{\alpha}$ and $V_{\alpha \sigma}$ are $4 \times 4$ matrices, $\Psi_{\sigma}^{\dagger}=\left(c_{1 \sigma}^{\dagger}, c_{2 \sigma}^{\dagger}, c_{3 \sigma}^{\dagger}, c_{4 \sigma}^{\dagger}\right)$ and $\Phi_{\sigma}^{\dagger}=$ $\left(a_{1 \sigma}^{\dagger}, a_{2 \sigma}^{\dagger}, a_{3 \sigma}^{\dagger}, a_{4 \sigma}^{\dagger}\right)$.

In the most general form (relaxed bath parametrization), the bath parameters are given by

$$
E_{B \sigma}^{\alpha}=\left(\begin{array}{cccc}
\varepsilon_{1 \sigma}^{\alpha} & 0 & 0 & 0 \\
0 & \varepsilon_{2 \sigma}^{\alpha} & 0 & 0 \\
0 & 0 & \varepsilon_{3 \sigma}^{\alpha} & 0 \\
0 & 0 & 0 & \varepsilon_{4 \sigma}^{\alpha}
\end{array}\right)
$$

and

$$
V_{\alpha \sigma}=\left(\begin{array}{cccc}
V_{11 \sigma}^{\alpha} & V_{12 \sigma}^{\alpha} & V_{13 \sigma}^{\alpha} & V_{14 \sigma}^{\alpha} \\
V_{21 \sigma}^{\alpha} & V_{22 \sigma}^{\alpha} & V_{23 \sigma}^{\alpha} & V_{24 \sigma}^{\alpha} \\
V_{31 \sigma}^{\alpha} & V_{32 \sigma}^{\alpha} & V_{33 \sigma}^{\alpha} & V_{34 \sigma}^{\alpha} \\
V_{41 \sigma}^{\alpha} & V_{42 \sigma}^{\alpha} & V_{43 \sigma}^{\alpha} & V_{44 \sigma}^{\alpha}
\end{array}\right)
$$

Within this representation, one have $2 *\left(N_{b}+N_{c} * N_{b}\right)$ free bath parameters to determine throughout the selfconsistent calculation (the factor 2 can be suppressed in the paramagnetic case, since $E_{B \uparrow}^{\alpha}=E_{B \downarrow}^{\alpha}$ and $\left.V_{\alpha \uparrow}=V_{\alpha \downarrow}\right)$. Alternatively, one can use a more efficient (constrained) bath parametrization [1, 2], which is faster and simpler to interpret:

$$
E_{B \sigma}^{\prime \alpha}=\left(\begin{array}{cccc}
\varepsilon_{\sigma}^{\alpha} & t_{B \sigma}^{\alpha} & t_{B \sigma}^{\prime \alpha} & t_{B \sigma}^{\alpha} \\
t_{B \sigma}^{\alpha} & \varepsilon_{\sigma}^{\alpha} & t_{B \sigma}^{\alpha} & t_{B \sigma}^{\prime \alpha} \\
t_{B \sigma}^{\prime \alpha} & t_{B \sigma}^{\alpha} & \varepsilon_{\sigma}^{\alpha} & t_{B \sigma}^{\alpha} \\
t_{B \sigma}^{\alpha} & t_{B \sigma}^{\prime \alpha} & t_{B \sigma}^{\alpha} & \varepsilon_{\sigma}^{\alpha}
\end{array}\right)
$$

and

$$
V_{\alpha \sigma}^{\prime}=\left(\begin{array}{cccc}
V_{\sigma}^{\alpha} & 0 & 0 & 0 \\
0 & V_{\sigma}^{\alpha} & 0 & 0 \\
0 & 0 & V_{\sigma}^{\alpha} & 0 \\
0 & 0 & 0 & V_{\sigma}^{\alpha}
\end{array}\right) .
$$

In the latter representation, we have explored the translational symmetry of the bath, considering that all the bath sites inside a given sub-bath have the same energy, and also that each cluster site hybridizes only with the correspondent site of the plaquette sub-bath. To compensate the simplification, we have included hopping between nearest-neighbor and nextnearest-neighbor sites within each sub-bath. In the simplified parametrization, we only have $2 *\left(4 * M_{b}\right)$ parameters to determine self-consistently, where $M_{b}=N_{b} / N_{c}$ is the number of sub-baths.

The parametrizations are related through the unitary ma$\operatorname{trix} S\left(S S^{T}=\mathbb{1}\right)$ which diagonalizes $E_{B \sigma}^{\prime \alpha}$, that is, $E_{B \sigma}^{\alpha}=$ $S E_{B \sigma}^{\prime \alpha} S^{T}$ and $V_{\alpha, \sigma}=S^{T} V_{\alpha \sigma}^{\prime}$, so that the bath function $\Delta(i \omega)=V^{T}\left(i \omega \mathbb{1}-E_{B}\right)^{-1} V$ remains invariant over the bath transformation.

Throughout this work, we have used the reduced bath parametrization to produce the phase diagrams displayed in Fig. 1. For some range of parameters, however, we have relaxed the bath parameters to confirm that our conclusions 
about the existence of different solutions and the stability of each one in different regions of the phase-diagram do not depend on the choose of the parametrization.

As an example, the converged bath parameters for the four relevant cases displayed at Fig. 5 can be seen on Table 1 Note that the results do not depend on the spin, since we concentrate on the paramagnetic solution. The eigenvalues of the diagonalized $E_{B \sigma}^{\prime \alpha}$ matrix are given by $\left\{\varepsilon^{\alpha}-t_{B}^{\prime \alpha}, \varepsilon^{\alpha}-t_{B}^{\prime \alpha}, \varepsilon^{\alpha}-\right.$ $\left.2 t_{B}^{\alpha}+t_{B}^{\prime \alpha}, \varepsilon^{\alpha}+2 t_{B}^{\alpha}+t_{B}^{\prime \alpha}\right\}$. For the four cases displayed on the table, we have the following set of eigenvalues: PGM, $\mathrm{U}=3.0, \mathrm{p}=0.06\{0.0768,0.0768,-0.1212,0.2548\}$; CFM, $\mathrm{U}=3.0, \mathrm{p}=0.05\{-0.039,-0.039,-0.033,0.031\}$; PGM, $\mathrm{U}=7.0, \mathrm{p}=0.06\{0.074,0.074,0.052,0.180\}$; CFM, $\mathrm{U}=7.0$, $\mathrm{p}=0.16\{-0.038,-0.038,-0.046,0.03\}$.

\begin{tabular}{|c|c|c|c|c|c|c|c|c|}
\hline \multirow{2}{*}{$\begin{array}{c}\text { Solution and } \\
\text { parameters: }\end{array}$} & \multicolumn{4}{|c|}{ Sub-bath 1} & \multicolumn{4}{c|}{ Sub-bath 2} \\
\cline { 2 - 10 } & $\varepsilon$ & $\mathrm{t}_{\mathrm{B}}$ & $\mathrm{t}_{\mathrm{B}}^{\prime}$ & $\mathrm{V}$ & $\varepsilon$ & $\mathrm{t}_{\mathrm{B}}$ & $\mathrm{t}_{\mathrm{B}}^{\prime}$ & $\mathrm{V}$ \\
\hline $\mathrm{PGM}, \mathrm{U}=3.0, \mathrm{p}=0.06$ & 0.718 & 0.094 & -0.005 & 0.874 & -0.399 & 0.047 & 0.016 & 0.608 \\
\hline $\mathrm{CFM}, \mathrm{U}=3.0, \mathrm{p}=0.05$ & -0.02 & 0.016 & 0.019 & 0.206 & 0.078 & -0.083 & -0.011 & 0.451 \\
\hline $\mathrm{PGM}, \mathrm{U}=7.0, \mathrm{p}=0.06$ & 0.095 & 0.032 & 0.021 & 0.249 & -0.088 & 0.013 & -0.004 & 0.316 \\
\hline CFM, U=7.0, $\mathrm{p}=0.16$ & -0.023 & 0.019 & 0.015 & 0.209 & 0.077 & -0.080 & -0.116 & 0.421 \\
\hline
\end{tabular}

TABLE I: Converged bath parameters for the auxiliary Anderson model within the constrained parametrization (Eqs. 5 and 6 .

A comparison between results obtained with the reduced (Eqs. 5 and 6) and relaxed (Eqs. 3 and 4) bath parametrizations can be seen on Fig. 1, which shows the double occupation probability and the imaginary part of the self-energy at the anti-nodal point, calculated on the imaginary axis. The results are for the half-filled case, with $t^{\prime}=0$. With both parametrizations, we can clearly distinguish the different solutions, CFM and PGM.
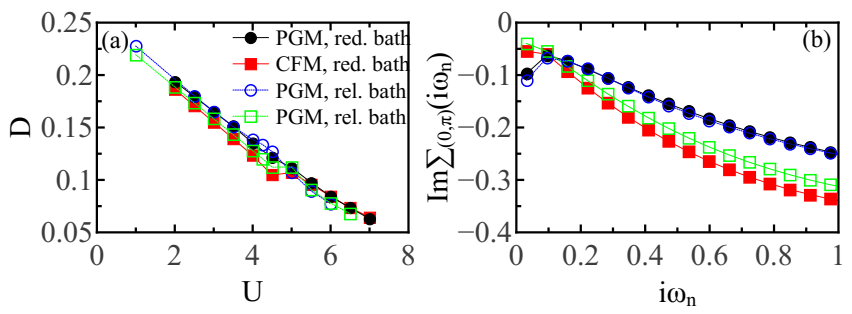

FIG. 1: Comparison between results obtained with different bath parametrization for the half-filled case with $t^{\prime}=0$. (a) Double occupation probability and (b) imaginary part of the self-energy at the anti-nodal point, calculated on the imaginary axis for $U=3.0$. Open symbols correspond to the relaxed bath parametrization (Rel. bath), while filled symbols were obtained with the constrained parametrization (Red. bath). With both bath parametrizations, we can distinguish two different solutions, a PGM (circles) and a CFM (squares).
Under the numerical calculation, the bath-parameters $\varepsilon^{\alpha}$, $t_{B}^{\alpha}, t_{B}^{\prime \alpha}$ and $V_{\alpha}$ are determined at each CDMFT-iteration by fitting the Anderson-impurity Weiss field with a $N_{b}$-pole bath function $\hat{\mathcal{G}}_{N_{b}}^{n e w}\left(i \omega_{n}\right)=i \omega_{n} \mathbb{1}-\hat{E}-\hat{\Delta}$, with $\Delta(i \omega)=$ $V^{T}\left(i \omega \mathbb{1}-E_{B}\right)^{-1} V$. The fitting is obtained via a conjugate gradient minimization algorithm, with a distance function that emphasizes the lowest frequencies [2, 3]

$$
f=\sum_{n} \sum_{i j} \frac{1}{\omega_{n}}\left|\mathcal{G}_{0}^{n e w}\left(\omega_{n}\right)-\mathcal{G}_{N_{b}}^{n e w}\left(\omega_{n}\right)\right|_{i j}
$$

The convergence criteria combines a convergence of the cluster Green's function between consecutive iterations with a small value of the distance function.

\section{II - LATTICE QUANTITIES IN MOMENTUM SPACE AND ENERGY CALCULATION}

As discussed in the main text, the cellular dynamical mean field calculation in a $2 \times 2$ plaquette cluster gives us frequency dependent quantities in specially symmetric points of the first quadrant of the Brillouin zone, $\vec{k}=(0, \pi),(\pi, 0),(\pi, \pi)$ and $(0,0)$. The reconstruction of the lattice quantities in a broader range of momentum can be obtained through a (truncated) Fourier expansion in which the cluster quantities are the expansion coefficients, that is,

$$
Q^{L}\left(k, i \omega_{n}\right)=\frac{1}{N_{c}} \sum_{i, j=1}^{N_{c}} Q_{i j}^{c}\left(i \omega_{n}\right) \exp \left[i \vec{k} \cdot\left(\vec{r}_{i}-\vec{r}_{j}\right)\right],
$$

where the sub-indices $L$ and $c$ stand for lattice and cluster quantities, respectively, and $\vec{r}_{i}$ and $\vec{r}_{j}$ are spacial intra-cluster coordinates.

Different periodization schemes have been proposed in the literature, related to different choices for the quantity $Q$, such as the self-energy scheme, $\Sigma$ [4], the cumulant scheme, $M=$ $\left[i \omega_{n}+\mu-\Sigma\right]^{-1}$ [5, 6], and the Green's function one [1, 7].

A comparison between results obtained with the three different procedures can be found in reference [8]; it is known that the choices $Q=M$ and $Q=G$ are equivalent in first order expansion and produce the best and the least clustersize-dependent results close to the Mott transition [9]. For this reason, we use the $M-$ scheme throughout this work.

In this case, we obtain the lattice cumulant from the cluster ones; the lattice self-energy and the lattice Green's function can then be obtained through the relations

$$
G^{L}\left(k, i \omega_{n}\right)=\left[M^{L}\left(k, i \omega_{n}\right)^{-1}-\varepsilon(k)\right]^{-1}
$$

where $\varepsilon(k)=-2 t\left(\cos k_{x}+\cos k_{y}\right)-4 t^{\prime} \cos k_{x} \cos k_{y}$ and

$$
\Sigma^{L}\left(k, i \omega_{n}\right)=i \omega_{n}+\mu-M^{L}\left(k, i \omega_{n}\right)^{-1} .
$$




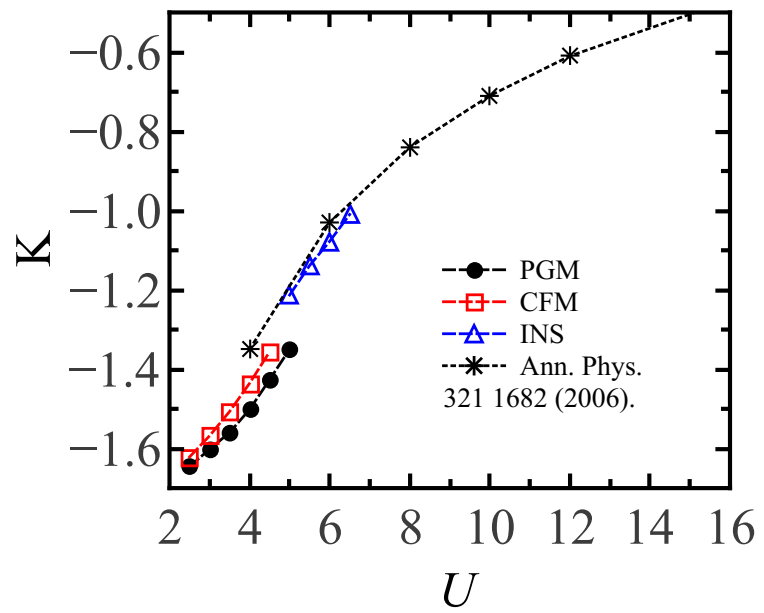

FIG. 2: Kinetic energy as a function of $U$ for half-filling and $t^{\prime}=$ 0.0. Comparison between our results and the one shown in reference [6].

Once we have $G^{L}\left(k, i \omega_{n}\right)$, we can calculate the kinetic energy with the relation

$$
K=\frac{1}{N_{k} \beta} \sum_{\sigma, k, n} \xi_{k} G\left(k, i \omega_{n}\right)
$$

where $\xi_{k}=\varepsilon_{k}-\mu$ is the electronic dispersion for the twodimensional square lattice, $N_{k}$ is the number of points in momentum space and $\beta=100 / t$ is the inverse effective temperature (used as a Matsubara grid in the ED-CDMFT implementation). For the paramagnetic solution, the summation over spin corresponds to a factor 2 . The summation over the Matsubara frequency, on the other hand, is more complicated and have to be done carefully in order to properly account for the tails for large frequency [10].

A comparison between our calculation of the kinetic energy and other present in the literature can be seen on Fig. 2, which shows $K$ as a function of $U$ for half-filling, $t^{\prime}=0.0$, and our three different solutions, that is, the CFM, PGM and Mott insulator.

The potential energy, on the other hand, is calculated through $U D$, where $D=\sum_{i}\left\langle n_{i \uparrow} n_{i \downarrow}\right\rangle$ is the double occupation probability.

The total energy $E=K+U D$, the potential $(U D)$, and the kinetic $(K)$ energies for the different solutions are displayed in Fig. 3 Panels (a)-(c) show the energy as a function of interaction for the half-filled case; panels (d)-(e) correspond to the doped case, for different values of interaction.

As discussed in the main text, when both CFM and PGM solution are present, the CFM is the stable solution (lower total energy). It is useful to separate the potential and kinetic energy contributions to the total energy. We observe that the PGM has a higher double occupation of sites $D$, i.e. a higher potential energy $U D$, and a lower (negative) kinetic energy
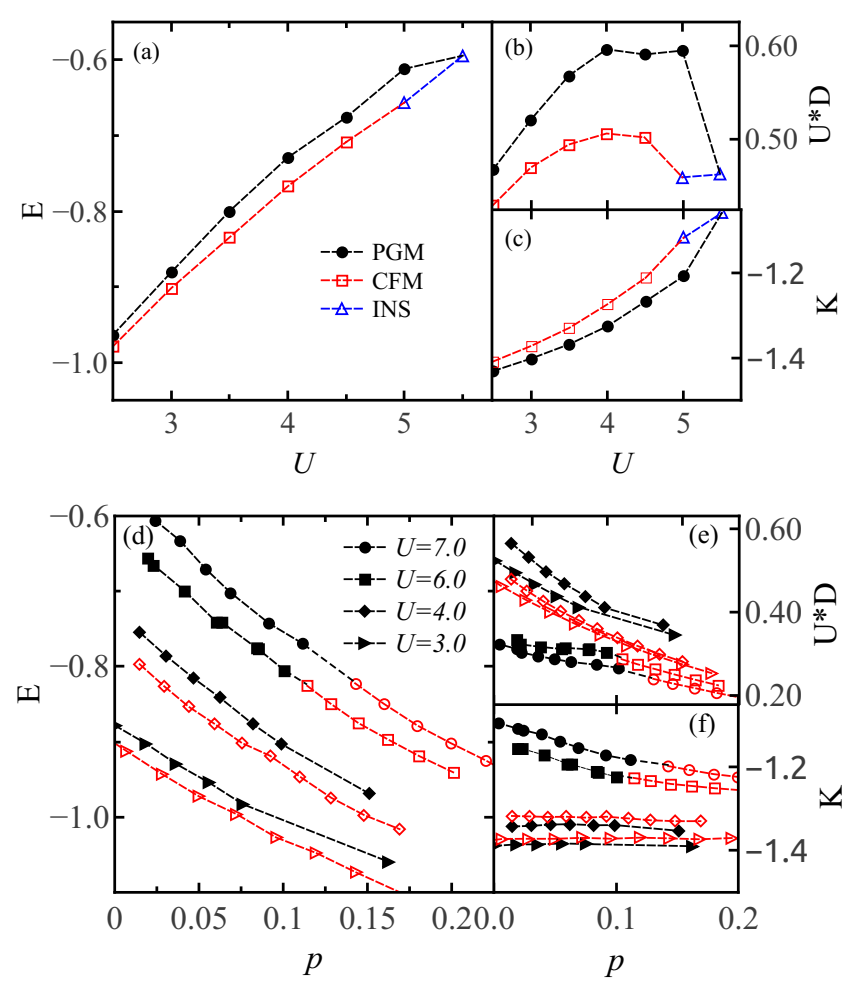

FIG. 3: Total, potential and kinetic energies (a)-(c) as a function of interaction at half-filling, and (d)-(e) as a function of doping for different interaction strengths. All the results were obtained with $t^{\prime}=-0.1$. Red-open symbols represent the CFM solution, while black-filled ones correspond to the PGM phase.

$K$ than the CFM. This is reminiscent of the Anderson's Resonating Valence Bond solution to the Heisenberg model [11], which favors a gain in kinetic energy with respect to the paramagnetic solution [12].

\section{III - SPECTRAL FUNCTION IN A BROADER FREQUENCY RANGE}
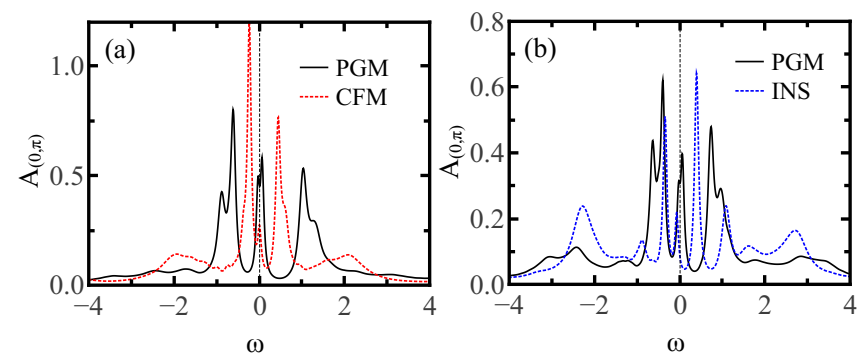

FIG. 4: Spectral function $A(\mathbf{k}, \omega)$ in the $\mathbf{k}=(0, \pi)$ point of momentum space for half-filling, $t^{\prime}=-0.1$, and different co-existing solutions, (a) CFM and PGM, for $U=4.0$ and (b) INS and PGM, for $U=5.0$. 
Fig. 4 shows the spectral function $A(\omega)_{(0, \pi)}$ at the antinodal point $\mathbf{k}=(0, \pi)$, at half-filling and $t^{\prime}=-0.1$, for $U=4.0$ and $U=5.0$ respectively. For $U=4.0$ one can observe a co-existence between a correlated Fermi metal and a pseudogap metal. For $U=5.0$, we find the pseudogap metal and a Mott insulator close to metal transition point $U_{c}$, displaying then a small gap. These are the same data shown on Fig. 2 of the main text, but with a larger frequency range. The Hubbard sub-bands are clearly visible in the insulator around $\omega=|U / 2|$ (Fig. 4.b), but their formation is already observable in the CFM (Fig. 4.a) and the PGM (Fig. 4.b).

\section{IV - RENORMALIZED ENERGIES AND FERMI SURFACE TOPOLOGY}

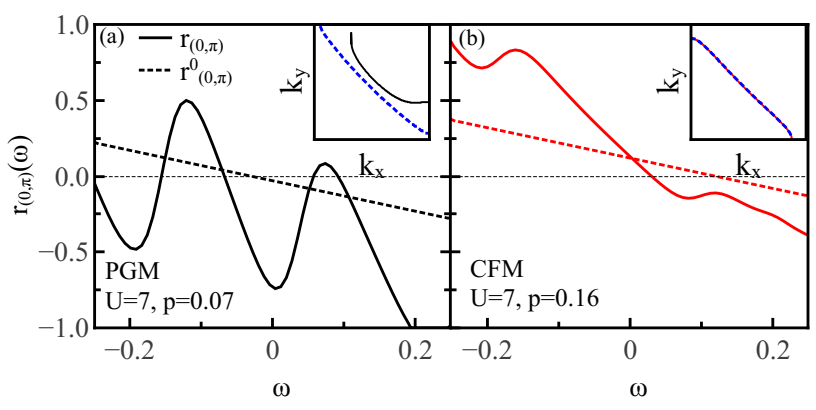

FIG. 5: Renormalized energies, $r_{(0, \pi)}(\omega)$ and $r_{(0, p i)}^{0}(\omega)$, at low frequency, calculated at the antinodal point, $\vec{k}=(0, \pi)$, for $t^{\prime}=-0.1$. Insets: Interacting Fermi surface (continuous line) compared with the non-interacting one (dashed line).

In order to see the effect of the correlation on the Fermi surface topology, one can consider the renormalized energy [6]:

$$
r_{k}(\omega)=-\operatorname{Re} G(k, \omega)^{-1}=\operatorname{Re} \Sigma_{k}(\omega)-\omega+\varepsilon_{k}-\mu .
$$

For a given $k$ in momentum space, the $k$-state is occupied if $r_{k}(0)<0$. If we consider then the cluster momentum $k=(0, \pi)$, a metallic solution has a hole-like Fermi surface if $r_{(0, \pi)}(0)<0$, while it has an electron-like Fermi surface if $r_{(0, \pi)}(0)>0$ (i.e. the $k=(0, \pi)$-state is empty)

In Fig. 5 we display $r_{(0, \pi)}(\omega)$ and compare it with $r_{(0, \pi)}^{0}(\omega)=-\omega+\epsilon_{(0, \pi)}-\mu_{0}$, where $\mu_{0}$ is the chemical potential of the non-interacting system $(U=0)$ which gives the same particle density.

Panel (a) shows the PGM for $U=7 t$ and rather small doping $p=0.07$, where the solution is the stable one. With respect to the $U=0$, one can observe that there is the pole contribution to the self-energy close to $\omega=0$ :

$$
\operatorname{Re} \Sigma_{k}(\omega) \simeq \mu_{0}+\frac{V^{2}}{\omega-\xi_{k}^{f}}
$$

with $\xi_{k}^{f}>0$, which pulls down $r_{k}(\omega=0)$ to more negative values than the non-interacting one $r_{k}^{0}(\omega=0)$. Therefore if one starts with a non-interacting Fermi surface at $U=0$ which is hole-like, and turns on $U$, the resulting interacting Fermi surface is even more hole-like (see inset of Fig 5]a). If one consider the Fermi-liquid CFM solution instead, displayed for $U=7 t$ and rather high doping $p=0.16$ where it is stable (panel b), the self-energy has no pole at low frequency, hence the effect of the interaction on the Fermi surface shape are much milder. In fact, for $p=0.16$, if one starts an electron-like Fermi surface for $U=0\left(r_{k}^{0}(\omega=0)>0\right)$, and then turns the interaction on, the resulting interacting Fermi surface remains electron-like (also $r_{k}(\omega=0)>0$ ). In this case the difference between the non-interacting and interacting Fermi surfaces is barely visible.

[1] S. S. Kancharla, B. Kyung, D. Sénéchal, M. Civelli, M. Capone, G. Kotliar, and A.-M. S. Tremblay, Anomalous superconductivity and its competition with antiferromagnetism in doped Mott insulators, Phys. Rev. B 77, 184516 (2008).

[2] M. Civelli, The doping-driven evolution of the superconducting state of a doped Mott insulator: a key for the high temperature superconductivity, Phys. Rev. B 79, 195113 (2009).

[3] M. Capone, M. Civelli, S. S. Kancharla, C. Castellani, and G. Kotliar, Cluster-dynamical mean-field theory of the densitydriven Mott transition in the one-dimensional Hubbard model, Phys. Rev. B 69, 195105 (2004).

[4] M. Civelli, M. Capone, S. S. Kancharla, O. Parcollet, and G. Kotliar, Dynamical Breakup of the Fermi Surface in a Doped Mott Insulator, Phys. Rev. Lett. 95, 106402 (2005).

[5] Tudor D. Stanescu and Gabriel Kotliar, Fermi arcs and hidden zeros of the Green function in the pseudogap state, Phys. Rev. B 74, 125110 (2006).

[6] Tudor D. Stanescu, Marcello Civelli, Kristjan Haule, Gabriel Kotliar, A cellular dynamical mean field theory approach to Mottness, Ann. Phys. (N.Y.) 321, 1682 (2006).

[7] David Sénéchal and A.-M. S. Tremblay, Hot Spots and Pseudogaps for Hole- and Electron-Doped High-Temperature Superconductors, Phys. Rev. Lett. 92, 126401 (2004).

[8] Marcello Civelli, Investigation of Strongly Correlated Electron Systems with Cellular Dynamical Mean Field Theory, $\mathrm{PhD}$ Thesis, Rutgers University, Piscataway, New Jersey, USA (2006), arXiv:0710.2802

[9] Shiro Sakai, Giorgio Sangiovanni, Marcello Civelli, Yukitoshi Motome, Karsten Held, Masatoshi Imada, Cluster-size dependence in cellular dynamical mean-field theory, Phys. Rev. B 85, 035102 (2012).

[10] Gerald D. Mahan, Many-Particle Physics, 3rd ed., Kluwer Academic/Plenum Publishers, New York, 2000.

[11] P. W. Anderson, The Theory of Superconductivity in the HighTc Cuprates, Princeton University Press, Princeton (1997).

[12] Luca F. Tocchio, Federico Becca, and Sandro Sorella, Hidden Mott transition and large- $U$ superconductivity in the twodimensional Hubbard model, Phys. Rev. B 94, 195126 (2016). 